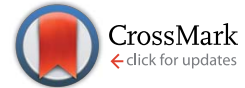

Cite this: RSC Adv., 2015, 5, 10211

Accepted 7th January 2015

DOI: $10.1039 / c 4 r a 16991 c$

www.rsc.org/advances
Received 24th November 2014

\section{A composite element bit design for magnetically encoded microcarriers for future combinatorial chemistry applications}

\author{
David M. Love, ${ }^{\star}$ Kunal N. Vyas, Amalio Fernández-Pacheco, Justin Llandro, \\ Justin J. Palfreyman, Thanos Mitrelias and Crispin H. W. Barnes
}

\begin{abstract}
We present a new composite element (CE) bit design for the magnetic bit encoding of suspended microcarriers, which has significant implications for library generation applications based on microfluidic combinatorial chemistry. The CE bit design consists of high aspect ratio strips with appropriate dipolar interactions that enable a large coercivity range and the formation of up to 14 individually addressable bits (16 384 codes) with high encoding reliability. We investigate $\mathrm{Ni}_{80} \mathrm{Fe}_{20}$ and $\mathrm{Co} \mathrm{CEs}$, which produce coercivity ranges of 8-290 Oe and 75-172 Oe, respectively, showing significant improvements to previously proposed bit designs. By maintaining the total magnetic volume for each CE bit, the barcode design enables a consistent stray field for in-flow magnetic read-out. The CE bit design is characterised using magneto-optic Kerr effect (MOKE) measurements and the reliability of the design is demonstrated in a multi-bit encoding process capable of identifying each bit transition for every applied magnetic field pulse. By constraining each magnetic bit to have a unique switching field using the CE design, we enable sequential encoding of the barcode using external magnetic field pulses. We therefore discuss how the new CE barcode design makes magnetically encoded microcarriers more relevant for rapid and noninvasive detection, identification and sorting of compounds in biomolecular libraries, where each microcarrier is for example capable of recording its reaction history in daisy-chained microfluidic splitand-mix processes.
\end{abstract}

\section{Introduction}

Combinatorial chemistry provides significant scaling advantages and has formed a cornerstone of medicinal chemistry over two decades ago. While modern drug discovery relies heavily on rational design and computer modelling, the use of combinatorial chemistry techniques is re-emerging ${ }^{1,2}$ in areas such as the formation of peptide libraries and formulation chemistry of drugs. Early efficiency estimates ${ }^{3}$ of combinatorial chemistry already showed that 1000 times more compounds can be generated for at least 600 times less the cost per compound compared to traditional methods, prompting pharmaceutical companies to adopt the technique in their drug development and study of related compounds. With modern economies of scale favouring automation and especially with the rise of microfluidic technologies this efficiency trend has since progressed exponentially. ${ }^{4,5}$ Here, we present advances in magnetic barcode design that make the use of suspended and magnetically encoded microcarriers (or tags) highly relevant for the development of future library generation and screening applications using lab-on-a-chip based combinatorial chemistry.

Cavendish Laboratory, Department of Physics, University of Cambridge, Cambridge CB3 OHE, UK. E-mail: david.michael.love@gmail.com
Previously, magnetic encoding of microcarriers was proposed for multiplexed point-of-care diagnostic applications, where nominally identical tags are encoded using coercivity tuned magnetic bits by externally applied magnetic field pulses, ${ }^{6,7,9-11}$ offering unique advantages in mass fabrication and scaling. The idea formed an unconventional approach ${ }^{12}$ to other suspension assay technologies, where carrier detection was typically based on nano-particle binding, ${ }^{13}$ optical barcodes ${ }^{14-16}$ or fluorescence. ${ }^{17,18}$ Coercivity tuning on the other hand, is achieved by patterning magnetic strips with different aspect ratios (Fig. 1a), giving each bit a unique field amplitude at which its magnetisation reversal occurs (i.e. switching between a 0 and 1 state) and allowing identical microcarriers to be uniquely encoded with a multi-bit code. It has been shown that batches of such encoded microcarriers can be functionalised with two different probe molecules on two distinct surfaces, ${ }^{19,20}$ using the incorporated thiol and epoxy surface groups. By also including control molecules on each microcarrier, a bioassay is capable of identifying true positives/negatives by probing the assay conditions using control targets purposely included in the analyte. When fluorescence of the target molecules indicates positive binding, the magnetically encoded microcarriers can be identified by the magnetic stray fields of their unique barcodes in-flow, as has previously been demonstrated ${ }^{8}$ using an 


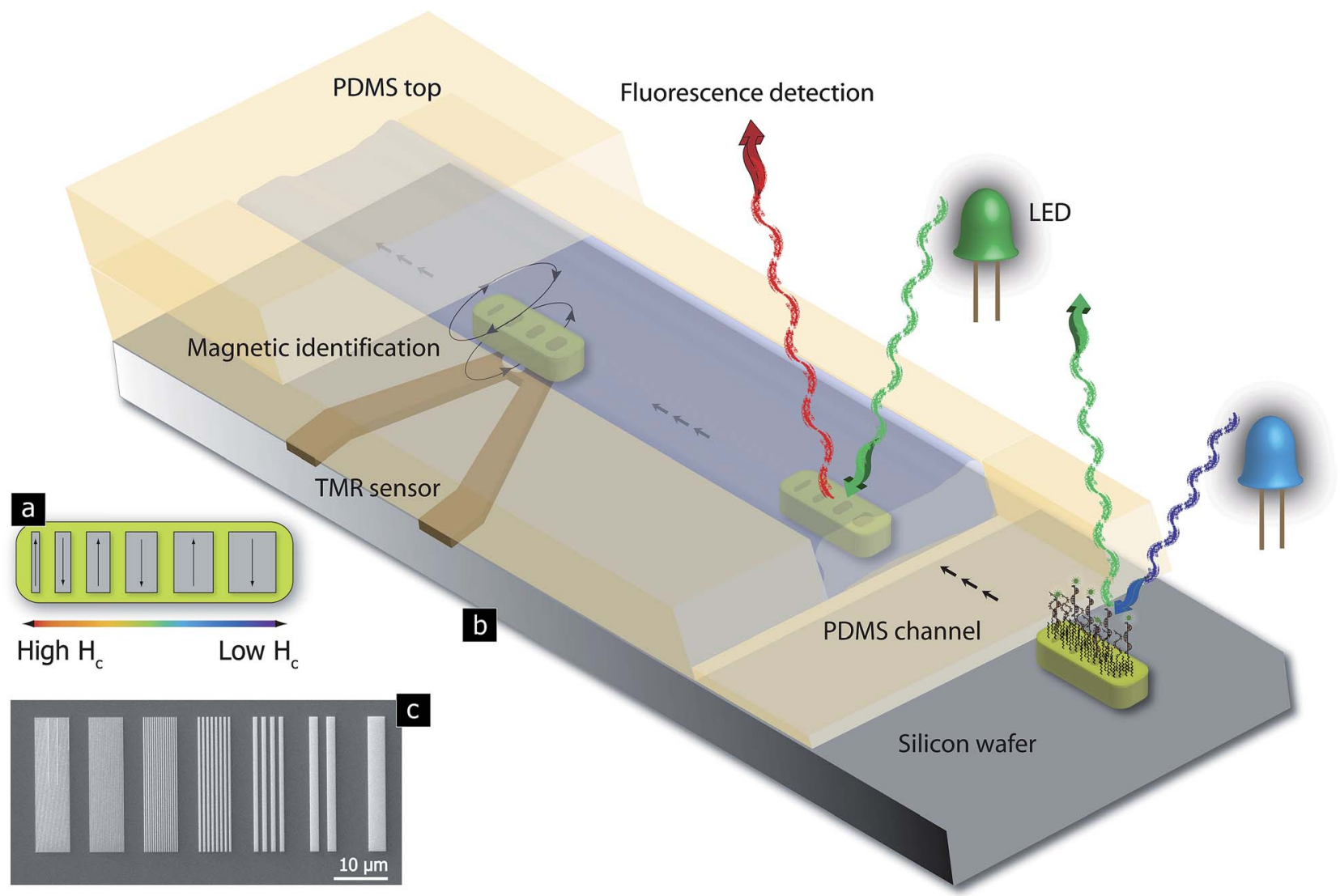

Fig. 1 (a) Illustration of the coercivity $\left(H_{c}\right)$ tuning technique and the original barcode design, ${ }^{6,7}$ where the coercivity distribution of 'bits' is determined by varying the aspect ratios of the ferromagnetic elements. (b) Illustration of the use of magnetically encoded microcarriers, with fluorescence detection and in-flow magnetic identification as previously demonstrated ${ }^{8}$ using polydimethylsiloxane (PDMS) moulded microfluidics with an integrated tunnelling magnetoresistance (TMR) sensor. (c) SEM image of the new composite element (CE) bit design, where each bit consists of high aspect ratio strips and maintains a consistent magnetic area.

integrated tunnelling magnetoresistance (TMR) sensor (illustrated in Fig. 1b).

However, the original bit design ${ }^{6,7}$ was prone to forming multi-domainal states, which heavily limited encoding reliability and therefore also the total number of distinct bits. Here, we expand on the concept of coercivity tuning and present a new composite element (CE) bit design (Fig. 1c) that is capable of encoding up to 14 bits (16 384 codes) with unique coercivity values to a high degree of accuracy. The CE bits consist of multiple strips with high aspect ratios and are engineered to enable a significantly wider coercivity distribution while maintaining sharp magnetisation reversal behaviour for reliable encoding. This makes the CE bit design highly relevant for the development of future microfluidics-based combinatorial chemistry applications, where each bit within a microcarrier can be individually encoded (when written from highest to lowest coercivity value) after each split-and-mix process. Compounds of interest could then be identified using multiplexed detection of the microcarriers' magnetic stray fields, all within a lab-on-a-chip platform. Here, we present the CE bit design and the intricacies that govern its encoding properties in order to demonstrate the reliability of the new barcode design.

\section{Composite element bit design}

As the magnetic barcodes are intended to be read out using a TMR sensor in-flow, we constructed a TMR-scanning microscope for detection optimisation. This instrument consists of an in-plane sensitive TMR head (Micro Magnetics Inc.) on a three axis nano-positioner (Thor Labs), which detects the inplane stray magnetic field components of patterned nanostructures. ${ }^{20}$ In single axis scans, inconsistencies in signal amplitude of identically encoded bit codes of the original design $^{6,7}$ were observed. The magnetic field and spatial resolution of the TMR sensor allows us to perform detailed 2D raster scans at set heights to map out the variation of magnetic stray field produced within the area of each element of the original design. Fig. 2 shows the real component $(X)$ of the sensor's impedance obtained from scanning a 3 bit magnetic tag containing Co elements with low aspect ratios. The $2 \mathrm{D}$ scan reveals information of both the amplitude and orientation of the magnetic fields produced by the elements along the sensor's sensitive direction, which is along the length of the elements. Domain formation within the low aspect ratio Co bits becomes apparent, particularly in the amplitude $(R)$ and phase $(\theta)$ data, since zero amplitude in $R$ indicates a domain wall while 

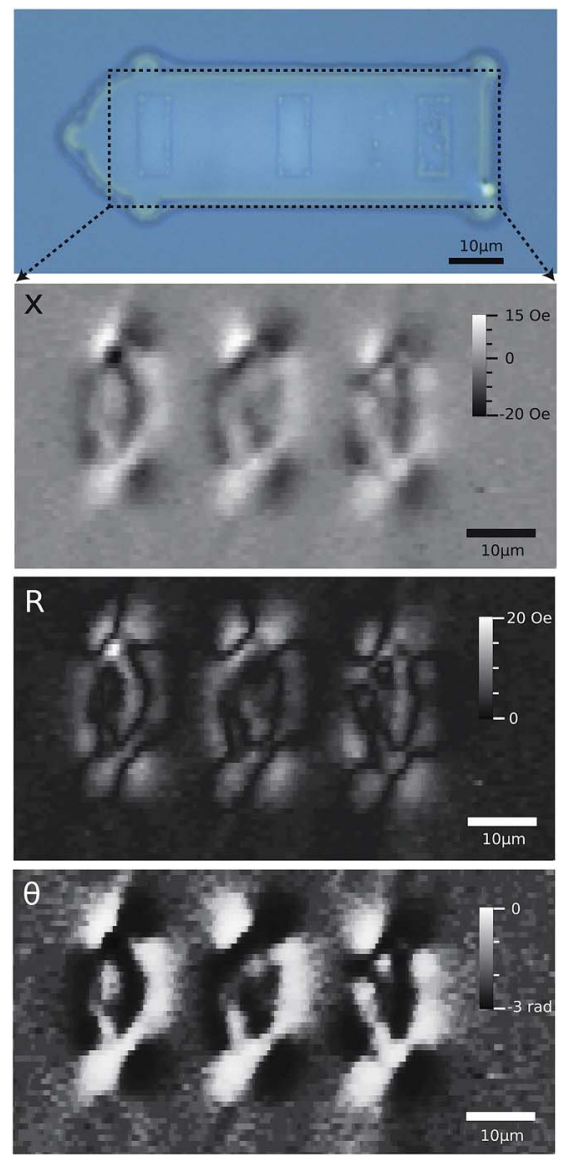

Fig. 2 2D stray field measurements of original magnetic bit design, ${ }^{6,7}$ obtained by scanning an in-plane TMR sensor with its sensitive direction along the length of the Co elements over a $50 \times 80 \mu \mathrm{m}$ area. Scan taken at a height of $2 \mu \mathrm{m}$ above the 3 bits with low aspect ratios. The images (top to bottom) show a microscope image of the microcarrier, the real component of the impedance sensor $(X)$, its amplitude $(R)$ and the phase $(\theta)$

opposite saturation in $\theta$ (and $X$ ) indicates the north and south poles of the magnetic domains.

It is evident that the domain breakdown observed for the low aspect ratio (low $H_{\mathrm{c}}$ ) bits reduces the stray field signal amplitude for in-flow detection and risks information loss. Although high aspect ratio (high $H_{\mathrm{c}}$ ) bits do not suffer from domain formation, their total magnetic volume is reduced, which limits the distance at which they can be detected inflow. ${ }^{8}$ Moreover, a domain breakdown prevents sharp switching and broadens the unique coercivity value of each bit, further increasing the risk of unintentional switching of neighbouring bits when writing a desired magnetic bit code using magnetic field pulses. ${ }^{6}$

The new CE bit design is able to overcome all these limitations by creating elements consisting of strips with significantly larger aspect ratios coupled by dipolar interactions. Here shape anisotropy is able to dominate the magnetic behaviour within each bit, as illustrated by the difference in domain formation between a single element and a CE bit in Fig. 3. As coercivity scales inversely with strip width, ${ }^{21,22}$ such a design is also able to

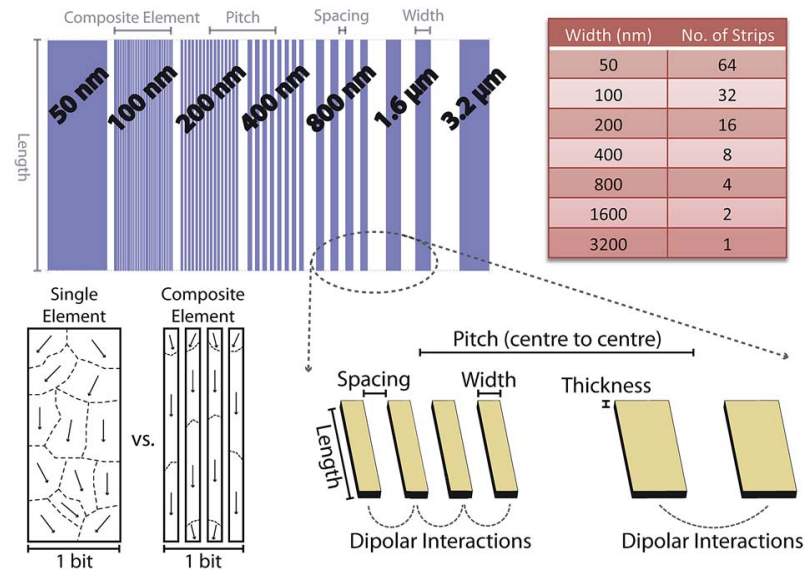

Fig. 3 The new CE bit design, where each CE bit contains the same magnetic volume and is comprised of a number of strips at set widths as outlined in the corresponding table. Extended section notes the length, thickness $(t)$, spacing $(s)$, width $(w)$ and pitch (element centre to centre) parameters used to characterise the possible coercivity distribution and switching behaviours. Diagram illustrates domain formation in original vs. CE bit design.

significantly widen the possible coercivity distribution, while the appropriate dipolar interactions and use of magnetically soft materials ensure sharp magnetisation reversal behaviour ${ }^{23}$ for reliable encoding. In the CE bit design the magnetic volume is maintained between bits by increasing the number of strips for CEs consisting of narrower strip widths, allowing for consistent magnetic read-out of each bit's stray field in-flow.

The in-plane easy axis coercivity $\left(H_{\mathrm{c}}\right)$ value of a patterned strips at set spacings is determined by ${ }^{22-24}$

$$
H_{\mathrm{c}}=\alpha\left(\frac{s}{w}\right) \times\left(\frac{t}{w}\right)+H_{0}
$$

where $t$ is the film thickness, $w$ the strip width, $s$ the strip spacing, $H_{0}$ a constant and $\alpha(s / w)$ a complex function for the dipolar interactions between strips, presented in detail by Adeyeye et $a l .{ }^{23}$ For a large coercivity range one would therefore select a relatively thick film (still within the single domain regime) with narrow strip widths compared to the strip lengths. ${ }^{22,24}$ In using large aspect ratios the coercivity values remain dominated by the $\mathrm{CE}$ width and thickness values. ${ }^{25}$ The spacing between strips within each CE can further be used to fine tune the element's coercivity and switching behaviour, by utilising the dipolar interactions between neighbouring strips. It is possible to adjust the onset and mode of the magnetisation reversal process ${ }^{26-29}$ at separations typically equal to or below the strip width.

For a maximised coercivity range we therefore select a relatively thick film of $15 \mathrm{~nm}$ for both the Co and $\mathrm{Ni}_{80} \mathrm{Fe}_{20}$ CEs to illustrate the difference between materials with and without crystalline anisotropy, respectively. The pitch between elements should be sufficiently large (e.g. $>5 \mu \mathrm{m})$ to prevent bits from interacting and to allow for in-flow stray field detection for set TMR sensors properties and rapid flow rates. ${ }^{8}$ By varying strip width, length and spacing we investigate their effects on the coercivity distribution and switching behaviour of the barcodes 
in order to optimise the CE bit design for reliable use in combinatorial chemistry based applications.

\section{Methods}

Samples were fabricated using e-beam patterning of a polymethyl-methacrylate (PMMA) resist bi-layer on a $3^{\prime \prime}$ Si wafer, comprised of a $100 \mathrm{~K}$ (1:2 PMMA : anisole diluted) bottom layer and $950 \mathrm{~K}$ (3:5 PMMA : anisole diluted) top layer. The lower molecular weight resist was spun at $2000 \mathrm{rpm}$ and soft baked at $180{ }^{\circ} \mathrm{C}$ for 90 seconds to give a $48 \mathrm{~nm}$ thickness measured on an ellipsometer. The higher molecular weight resist was subsequently dispensed and spun with maximum acceleration at $2700 \mathrm{rpm}$ and soft baked at $180^{\circ} \mathrm{C}$ for 90 seconds to give a combined thickness of $77 \mathrm{~nm}$. To prevent significant inter-diffusion between resist layers, the second coating was quickly dispensed using a $10 \mathrm{ml}$ syringe and immediately spun, as otherwise the solvent from the $950 \mathrm{~K}$ resist would penetrate the first layer within the order of one second.

Patterns for the CEs were designed using CleWin 4 (WieWeb software v.o.f.) and exposed using a Leica VB6 UHR e-beam writer at a $100 \mathrm{kV}$ beam voltage, and developed in a $5: 15: 1$ ratio of methyl-isobutyl-ketone : isopropanol : methyl-ethylketone (MIBK : IPA : MEK) for 5 seconds followed by a thorough IPA rinse and $\mathrm{N}_{2}$ gas dry. The $\mathrm{Co}(99.95 \%)$ and $\mathrm{Ni}_{80} \mathrm{Fe}_{20}(99.97 \%)$ sources were purchased from Goodfellow Ltd. and were both thermally deposited, at a rate of $0.025 \mathrm{~nm} \mathrm{~s}^{-1}$, using an Edwards Auto306 evaporator with a base pressure of $2 \times 10^{-7} \mathrm{mBar}$ to a thickness of $15 \mathrm{~nm}$, each with a $5 \mathrm{~nm}$ Au (99.99\%) capping layer to prevent oxidation. All growth heights were confirmed using atomic force microscopy (AFM). The hysteresis loops were measured by longitudinal magneto-optic Kerr effect ${ }^{30}$ (MOKE) on elements with appropriate pitch using a NanoMOKE magnetometer, produced by Durham Magneto Optics Ltd., capable of focusing the laser down to a $5 \mu \mathrm{m}$ spot diameter. Each presented MOKE coercivity value was obtained by averaging 200 hysteresis loops measured on each of at least 16 identical barcodes.

\section{Coercivity tuning}

The MOKE characterisation of the $\mathrm{Ni}_{80} \mathrm{Fe}_{20}$ and Co CE bits show a wide coercivity range, effectively increasing the operation margin of the bit design by utilising narrower strip dimensions. Fig. 4 [top] shows the average coercivity values plotted against strip widths, for bit designs where strip spacings are equal to strip widths, revealing a coercivity range of 8-290 Oe for $\mathrm{Ni}_{80} \mathrm{Fe}_{20}$ and 75-172 Oe for Co. These ranges are significantly wider than the 27-60 Oe for $\mathrm{Ni}_{80} \mathrm{Fe}_{20}$ and 32-120 Oe for Co elements reported for the original bit design. ${ }^{7}$ The magnetically soft $\mathrm{Ni}_{80} \mathrm{Fe}_{20}$ elements are clearly preferable and were also measured at two lengths, $25 \mu \mathrm{m}$ and $10 \mu \mathrm{m}$, and plotted on top of each other showing no significant variation. This behaviour was expected due to the extreme aspect ratios within the CE bit design, causing the length demagnetisation factor to be suppressed, and leaving the elements' demagnetisation value determined predominantly by the width and thickness values. ${ }^{25}$
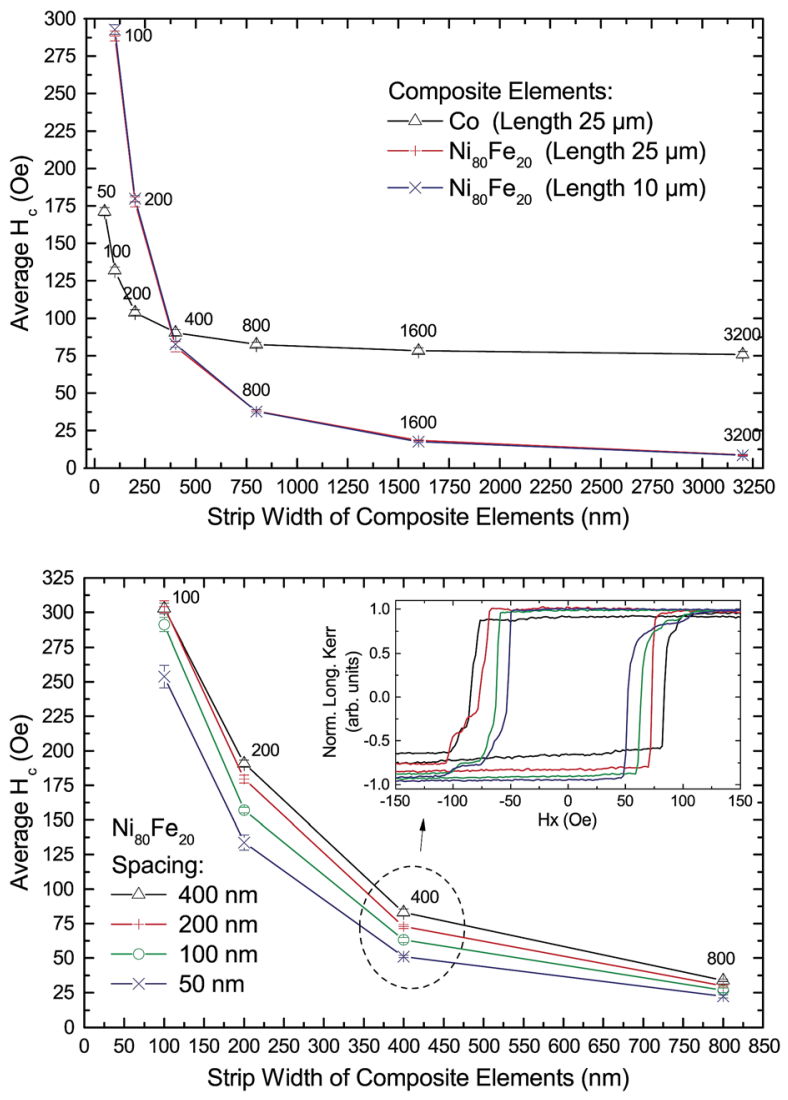

Fig. 4 [Top] Coercivity range, obtained using longitudinal MOKE, of $\mathrm{Co}\left(25 \mu \mathrm{m}\right.$ length) and $\mathrm{Ni}_{80} \mathrm{Fe}_{20}(10 \mu \mathrm{m}$ and $25 \mu \mathrm{m}$ lengths) $\mathrm{CE}$ bits with strip widths varying between 50-3200 nm and 100-3200 nm, respectively. The spacing between strips within each $C E$ is equal to the strip width. [Bottom] Coercivity values obtained by varying the spacing between strips of element widths $800 \mathrm{~nm}, 400 \mathrm{~nm}, 200 \mathrm{~nm}$ and $100 \mathrm{~nm}$ each by $400 \mathrm{~nm}, 200 \mathrm{~nm}, 100 \mathrm{~nm}$ and $50 \mathrm{~nm}$. Inset of switching behaviour (hysteresis loops) of the $400 \mathrm{~nm} \mathrm{Ni}_{80} \mathrm{Fe}_{20}$ element at 50-400 $\mathrm{nm}$ spacings. Every averaged data point represents the mean of 16 replica measurements of the same element geometry, with the error bars showing the $\pm 1 \sigma$ variation in coercivity. Point labels represent values along the $x$-axis.

The spacing between strips can itself further effect a CE's coercivity value and switching behaviour. Fig. 4 [bottom] shows $\mathrm{Ni}_{80} \mathrm{Fe}_{20}$ elements with strip widths between $100-800 \mathrm{~nm}$, each varying by spacings of $50-400 \mathrm{~nm}$. It becomes evident that decreasing the separation between strips decreases the overall element's coercivity $\left(H_{\mathrm{c}}\right)$ while maintaining consistently sharp switching behaviour, as seen in the inset hysteresis loops of elements with $400 \mathrm{~nm}$ strip widths. The magnetostatic coupling between strips is determined by the number of strips, their spacing and width, and will effect both the overall coercivity as well as the mode by which an element reverses. This can range from a sudden avalanche-like switch to a more gradual cascading of pairs of coupled antiparallel strips. ${ }^{26-28}$ In the later case, systems with stronger dipolar interactions, a closer spacing between strips can actually increase the element's coercivity, ${ }^{29}$ since the magnetisation reversal onset is due to domain formation at defects while the transition itself is a slow 
cascade-like switching of strongly interacting strips, resulting in a slanted hysteresis loop.

However, the varied strip spacings of the CE bit designs have sufficiently low coupling to give an earlier reversal onset at reduced separations due to a lower domain formation barrier. Domains typically occur at the strip ends and rapidly propagate through all neighbouring strips without forming antiparallel intermediate states during the reversal process. The fine tuning of the dipolar interactions at the higher coercivity elements becomes increasingly difficult, as a greater number of strips (to preserve the total magnetic volume between elements) tends to result in more gradual transitions. For our final CE bit design we select spacings equal to the strip width to ensure appropriate dipolar interaction strengths.

The benefits of using magnetically soft materials for coercivity tuning are further illustrated in Fig. 5, where stacked MOKE loops of Co and $\mathrm{Ni}_{80} \mathrm{Fe}_{20}$ elements reflect the coercivity range presented in Fig. 4. Crucial for reliable magnetic bit encoding are sharp transitions where magnetisation is dominated by shape anisotropy, reflected by sharp changes in Kerr signal. The Co elements produce rounded and overlapping loops that can send neighbouring elements into minor loop positions or even full transitions and therefore heavily limit writing reliability, as was noted for the original bit design. ${ }^{6,7}$ The
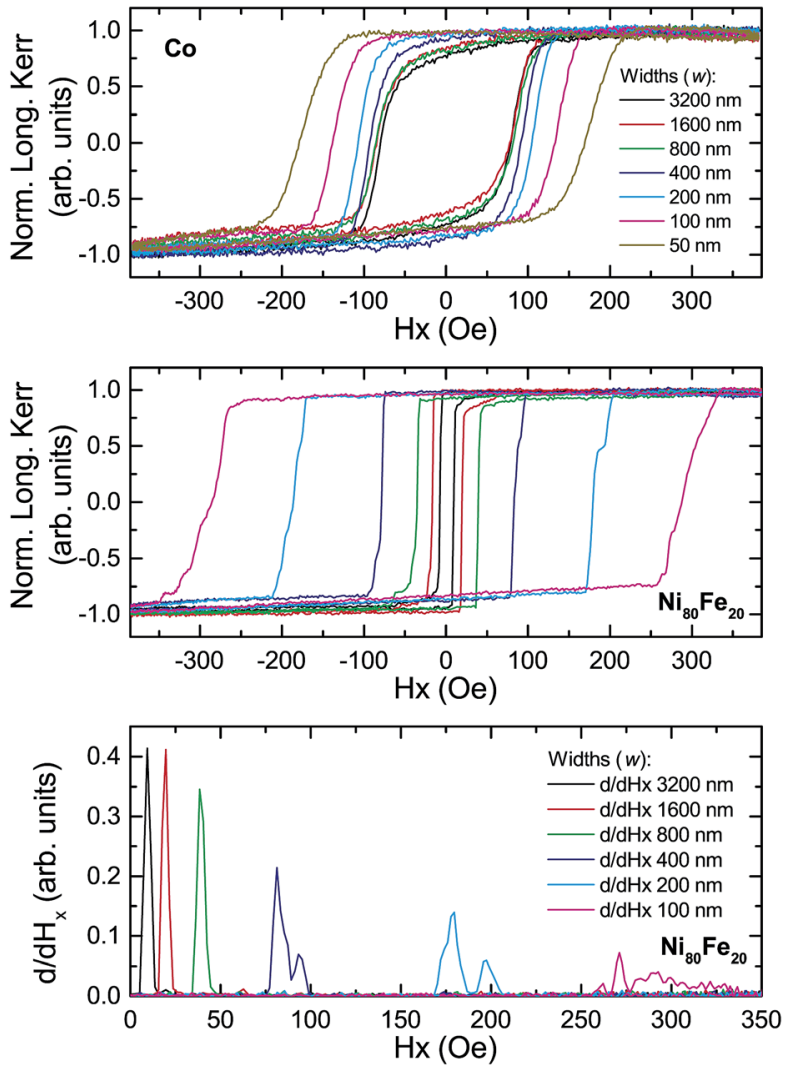

Fig. 5 Longitudinal focused MOKE measurements of CE bits where strip width equals strip spacing. [Top] Hystereses of Co CEs varying in widths $50-3200 \mathrm{~nm}$. [Middle] Hysteresis of $\mathrm{Ni}_{80} \mathrm{Fe}_{20}$ CEs varying in widths 100-3200 nm. [Bottom] Illustration of distinct switching in differential plot of positive half of $\mathrm{Ni}_{80} \mathrm{Fe}_{20} \mathrm{CE}$ hysteresis.
$\mathrm{Ni}_{80} \mathrm{Fe}_{20}$ elements on the other hand, result in much sharper transitions that are well separated from one another. This is particularly evident in the bottom plot of Fig. 5, where the derivatives of the $\mathrm{Ni}_{80} \mathrm{Fe}_{20}$ CE Kerr signals show no overlap between bits. The broadening of the differential plots for high $H_{\mathrm{c}}$ elements comprised of narrower strips, corresponding to more slanted hysteresis loop, is due to the greater difficulty of balancing dipolar interactions for growing arrays of strips, due to the tendency of forming antiparallel states during the reversal process. With growing arrays of strips there is also an increased likelihood of dispersion in CE strip features, where edge effect and lithography defects become more relevant in the reversal process. Nonetheless, it is evident that it is essential to use magnetically soft materials for the CE bit design to ensure reliable encoding of large bit codes.

\section{Magnetic bit encoding}

The reliable encoding process of the CE bit design can be demonstrated via MOKE by intentionally de-focusing the laser to illuminate multiple bits simultaneously to show compounded transitions of the elements characterised in Fig. 4 and 5. Fig. 6 shows the distinct transitions of three neighbouring elements in one MOKE loop, with strip widths of $200 \mathrm{~nm}$, $400 \mathrm{~nm}$ and $800 \mathrm{~nm}$ resulting in transitions at $177 \mathrm{Oe}, 81$ Oe and 38 Oe, respectively. By placing the laser slightly off-centre between the three bits the measured changes in Kerr amplitude are proportional to the level of illumination, allowing for the identification of the switching bit both from its change in Kerr signal as well as its coercivity value. By then decreasing the applied field amplitude accordingly, either all three, two, one or no bits can be forced to flip.

Fig. 6 can now be used as a reference for an encoding process, as the different amplitude changes in Kerr signal of the de-focused laser position can be used to precisely identify each switching bit of the three element system. Fig. 7 demonstrates such an encoding process, where a 101 bit code is written from a 000 initial configuration. The plots show all transitions that the three bit system undergoes for every applied field pulse, thereby locking in the desired bit states from highest to lowest coercivity value with decreasing field amplitude. The orange $\mathrm{X}$ indicates when the field sequence has been completed and the desired 101 bit code is written. The schematic of Fig. 7 illustrates the same process, where the number of intermediate transitions decreases with decreasing applied field amplitude. The encoding process is carried out at $1 \mathrm{~Hz}$ due to induction limitations of our electromagnet, whereas the bit encoding itself can be performed much quicker (i.e. $\mathrm{MHz}$ range) and is only limited by domain formation and motion. ${ }^{31}$ The de-focused MOKE technique can also be used to demonstrate encoding of longer bit codes, but visually distinguishing all individual Kerr amplitude changes becomes increasingly difficult.

\section{Discussion}

This investigation aims to demonstrate that the CE bit design, constructed with magnetically soft materials and appropriate 

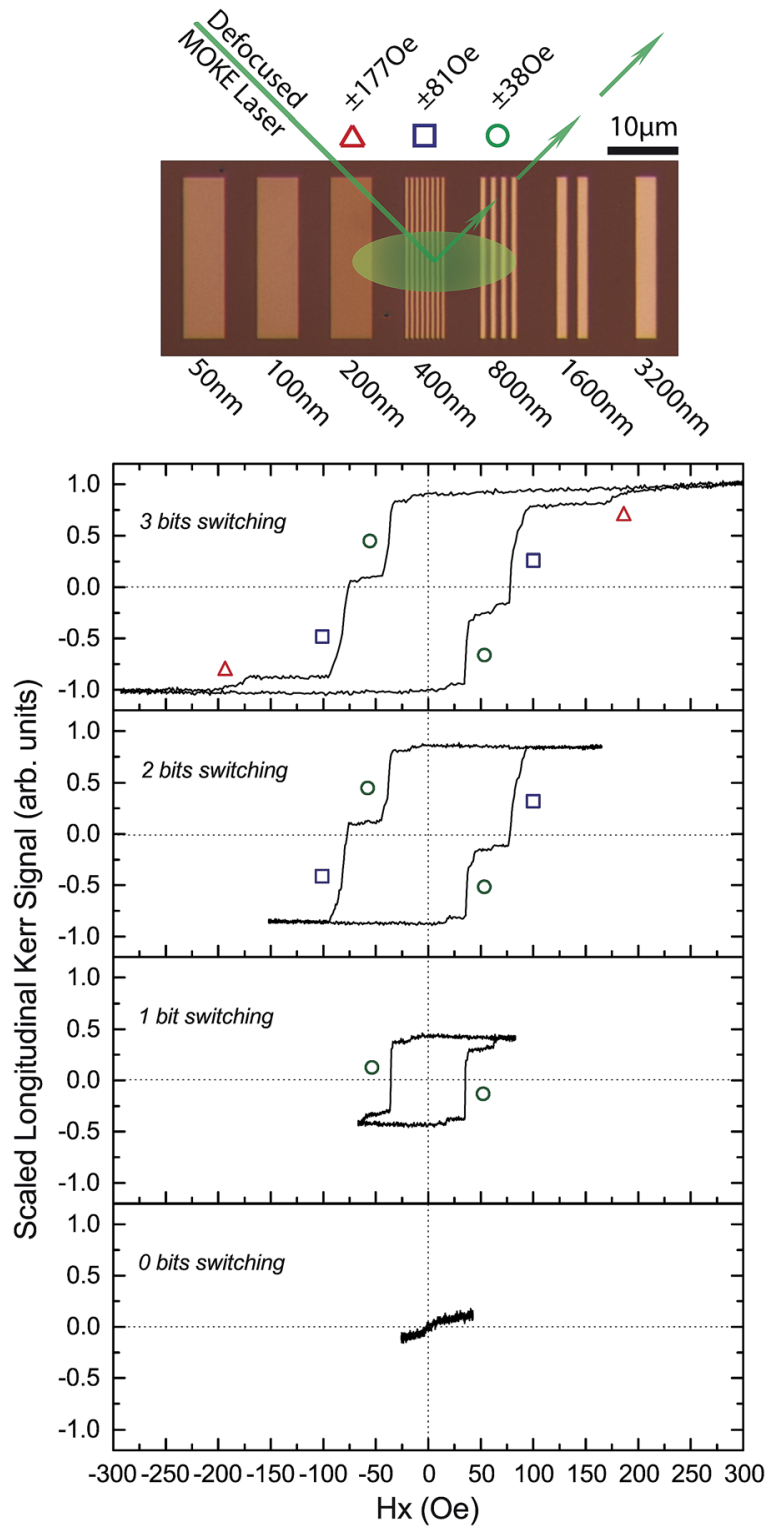

Fig. 6 De-focused MOKE measurements showing distinct switching of 3 neighbouring $\mathrm{Ni}_{80} \mathrm{Fe}_{20} \mathrm{CE}$ bits with strip widths $200 \mathrm{~nm}$ (177 Oe), $400 \mathrm{~nm}(81 \mathrm{Oe})$ and $800 \mathrm{~nm}(38 \mathrm{Oe})$, where strip spacing equals strip width. The laser is intentionally de-focused to illuminate all 3 bits, but placed off-centre to bias the Kerr signal, causing distinguishable amplitude changes corresponding to the switching of each of the 3 bits. As annotated on the microscope image, the illumination is proportional to change in Kerr signal, where the element with $400 \mathrm{~nm}$ strip widths sees the most change and the element with $200 \mathrm{~nm}$ strip widths sees the least. In varying the field intensity produced by the electromagnet at $1 \mathrm{~Hz}$ the switching of 3 bits, 2 bits, 1 bit and no bits is observed. All plots are averaged over 100 measurements and scaled relative to each other in Kerr signal by proportional change of $\Delta$ intensity/lintensityl to the top plot with maximum field amplitude.

dipolar interactions, enables a wide coercivity range for reliable encoding without compromising read-out properties. This makes the CE barcode and magnetically encoded microcarriers highly applicable for future combinatorial chemistry applications. While Fig. 4 suggests that up to 20 bits (1 048576 codes) at $\pm 1 \sigma$ separation could be achieved using $\mathrm{Ni}_{80} \mathrm{Fe}_{20}$ CEs ranging in strip widths between $50-3200 \mathrm{~nm}$, this measurement is of individual bits and does not take into account the effects of field pulses on neighbouring bits. The differential plot of Fig. 5 limits the true number of uniquely addressable bits as it is important to not send neighbouring bits into partially switched states and risk detection inconsistencies, which becomes increasingly difficult at smaller widths. We therefore make a more rigorous estimate of up to 14 uniquely addressable bits (16 384 codes) based on non-overlapping regions of Fig. 5 for widths 50-3200 nm using $\mathrm{Ni}_{80} \mathrm{Fe}_{20}$ for the CE bit design. However in theory, by using more complex sensor arrays in-flow with rigorous signal processing, it is also possible to envisage longer bit codes in the future, as demonstrated by developments ${ }^{32}$ in the magnetic hard disk drive industry.

The scaling advantages of bit encoding for library generation using combinatorial chemistry can be modelled by a Poisson distribution using the assumptions of consistently even (50:50) split-and-mix processes, a $6 \sigma$ accuracy in each chemistry step and a $2 \sigma$ accuracy in every encoding step. This gives a joint failure probability of $P=1.05 \times 10^{-6}$ for which we can find the appropriate level of degeneracy $(\lambda)$, indicating the average number of tags per end state that are required at the onset to guarantee that every split-and-mix compound and bit code is realised in the assay. Using the equation ${ }^{33} P=1-\left(1-\mathrm{e}^{-\lambda}\right)^{2^{n}}$ where $\lambda=N / 2^{n}$, we can determine the total number of tags $(N)$ required for a set number of combinatorial chemistry and bit encoding steps $(n)$. Table 1 illustrates the exponential scaling advantages in library generation with the linear scaling of splitand-mix steps. Therefore, using 384561 tags (with 14 bits) in a 14 step split-and-mix process will ensure that at least one or more tag carries every possible compound (16 384 in total) with the correct bit code. Encoding in-flow is also possible since the time scales for Néel rotation ${ }^{34}$ are many orders of magnitude below that of Brownian motion.

Since a $3^{\prime \prime}$ Si wafer can hold several million microcarriers $(100 \times 20 \mu \mathrm{m}$ footprint $)$ the number of required microcarriers can easily be met at low cost. Mass fabrication of feature sizes down to $50 \mathrm{~nm}$ can already be accomplished by existing semiconductor lithography procedures, such as interference lithography. Based on material and running costs of the bi-functional microcarrier fabrication procedure ${ }^{19}$ we estimate a cost of $\$ 100$ for 4 million tags on a $3^{\prime \prime}$ wafer, which can be further reduced when using industrial wafer sizes.

As the true advantage of magnetically encoded microcarriers is scalable library generation and not rapid diagnostics, compared to for instance nanoparticle-based applications, the relatively large size of the microcarriers is not a limiting factor. Since the area of the tags is comparable to the spot sizes used in printed microarrays, optically intensive techniques such as fluorescence detection can still be used to perform quantitative analysis. Multiplexing can then rapidly identify compounds of interest based on the magnetic stray fields of the microcarriers' barcodes in-flow. ${ }^{33}$ For an alternative to fluorescence detection, it has also been shown ${ }^{35}$ that it is possible to incorporate $\mathrm{Au}$ gratings on micron sized carriers to detect binding interactions using grating-coupled surface plasmon resonance (GCSPR). 

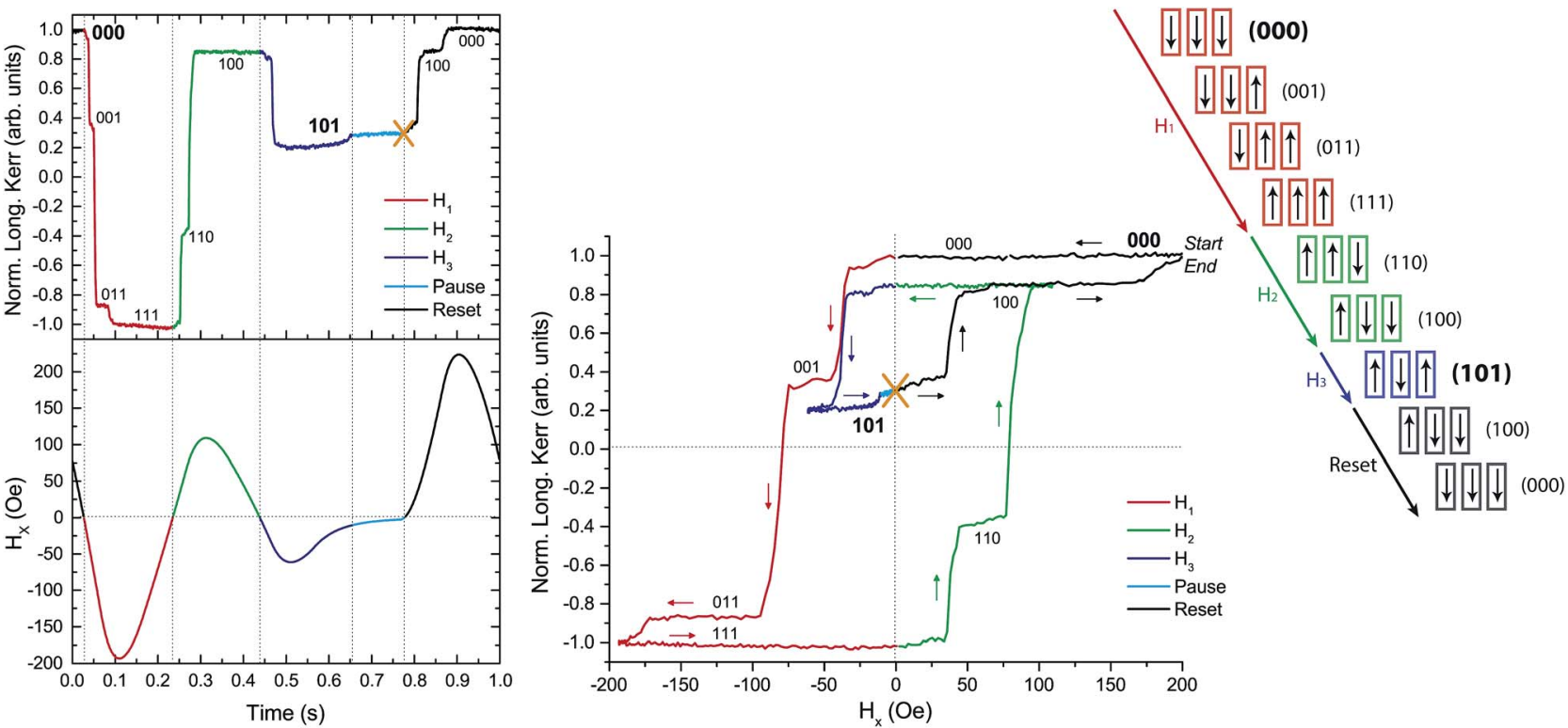

Fig. 7 Demonstration of controlled encoding of a 101 bit code from a 000 initial configuration in three neighbouring $\mathrm{Ni}_{80} \mathrm{Fe}_{20} \mathrm{CE}$ bits using defocused longitudinal MOKE, where distinguishable amplitude changes in Kerr signal correspond to the magnetisation reversal of each bit (as illustrated in Fig. 6). [Left] Normalised variation of Kerr signal with respect to applied field over time (at $1 \mathrm{~Hz}$ ), showing required sequence of switches to write the desired 101 code. [Right] Hysteresis loop of the same encoding process, with a corresponding animation of the three illuminated bits undergoing all switching steps for each applied field pulse. The orange $X$ indicates when the field sequence is completed and the desired 101 bit code is written.

Table 1 Scaling of compound generation using combinatorial chemistry, where the degeneracy and minimum number of tags required are estimated using the assumptions of even split-and-mix, $6 \sigma$ chemistry and $2 \sigma$ encoding accuracies

\begin{tabular}{llll}
\hline Steps $(n)$ & Num. compounds & Degeneracy $(\lambda)$ & Num. tags $(N)$ \\
\hline 1 & 2 & 14.5 & 29 \\
5 & 32 & 17.2 & 552 \\
10 & 1024 & 20.7 & 21196 \\
14 & 16384 & 23.5 & 384561
\end{tabular}

\section{Conclusion}

We have presented in detail the characteristics which govern the magnetic behaviour and reliability of the new CE bit design in order to demonstrate that coercivity tuned multi-bit encoding is feasible for large bit codes. The appropriate design of grouped high aspect ratio strips enables a large operation margin for coercivity tuning, with high encoding reliability and without limiting read-out properties. While the successful functionalisation of the epoxy and thiol surface groups on a single microcarrier has already been demonstrated, ${ }^{11,20}$ the proposed tagging technology can be extended to incorporating other well established surface chemistry processes, by for example adding a sputtered silicon dioxide coating. As the barcode and microcarrier designs are highly adaptable, magnetic encoding of microcarriers using the $\mathrm{CE}$ bit design has broad implications for the lab-on-a-chip community, with a wide range of potential applications relevant to the plethora of existing libraries of biomolecules which would benefit from a rapid method of compound detection, identification and sorting.

\section{Acknowledgements}

The authors gratefully acknowledge the EPSRC for financial support and thank Dr Andrew Thompson for valuable discussions. DL, TM and CHWB acknowledge the Cambridge Philosophical Society, the Lundgren Research Award, the Development of Prototype Grant (Innovate UK), the Brian Mercer Feasibility Award (Royal Society) and Cambridge BioMagnetics Ltd. AFP acknowledges the Winton Programme for the Physics of Sustainability.

\section{References}

1 T. Kodadek, Chem. Commun., 2011, 47, 9757-9763.

2 Y. Gao and T. Kodadek, Chem. Biol., 2013, 20, 360-369.

3 A. Persidis, Nat. Biotechnol., 1998, 16, 691-693.

4 P. S. Dittrich and A. Manz, Nat. Rev. Drug Discovery, 2006, 5, 210-218.

5 P. Watts and S. J. Haswell, Curr. Opin. Chem. Biol., 2003, 7, 380-387.

6 J. R. Jeong, J. Llandro, B. Hong, T. J. Hayward, T. Mitrelias, K. P. Kopper, T. Trypiniotis, S. J. Steinmuller, G. K. Simpson and J. A. C. Bland, Lab Chip, 2008, 8, 18831887.

7 B. Hong, T. J. Hayward, J. R. Jeong, J. F. K. Cooper, J. J. Palfreyman, T. Mitrelias, A. Ionescu, J. A. C. Bland and C. H. W. Barnes, J. Appl. Phys., 2009, 105, 034701. 
8 K. N. Vyas, B. Hong, J. F. K. Cooper, J. J. Palfreyman and C. H. W. Barnes, IEEE Trans. Magn., 2011, 47, 1571-1574.

9 T. Mitrelias, J. F. K. Cooper, K. N. Vyas, J. J. Palfreyman, B. Hong, T. J. Hayward and C. H. W. Barnes, J. Appl. Phys., 2010, 107, 09B319.

10 T. J. Hayward, B. Hong, K. N. Vyas, J. J. Palfreyman, J. F. K. Cooper, Z. Jiang, J. R. Jeong, J. Llandro, T. Mitrelias, J. A. C. Bland and C. H. W. Barnes, J. Phys. D: Appl. Phys., 2010, 43, 175001.

11 J. J. Palfreyman, P. Beldon, B. Hong, K. N. Vyas, J. F. K. Cooper, T. Mitrelias and C. H. W. Barnes, 8th International Conference on the Scientific and Clinical Applications of Magnetic Carriers, 2010, pp. 184-191.

12 J. Llandro, J. J. Palfreyman, A. Ionescu and C. H. W. Barnes, Med. Biol. Eng. Comput., 2010, 48, 977-998.

13 D. M. Bruls, T. H. Evers, J. A. H. Kahlman, P. J. W. van Lankvelt, M. Ovsyanko, E. G. M. Pelssers, J. J. H. B. Schleipen, F. K. de Theije, C. A. Verschuren, T. van der Wijk, J. B. A. van Zon, W. U. Dittmer, A. H. J. Immink, J. H. Nieuwenhuis and M. W. J. Prins, Lab Chip, 2009, 9, 3504-3510.

14 W. Z. Ho, J. Collins and C. J. Hou, US Pat. 12/832,972, 2011. 15 D. C. Pregibon, M. Toner and P. S. Doyle, Science, 2007, 315, 1393-1396.

16 H. Lee, J. Kim, H. Kim, J. Kim and S. Kwon, Nat. Mater., 2010, 9, 745-749.

17 S. A. Dunbar, C. A. Vander Zee, K. G. Oliver, K. L. Karem and J. W. Jacobson, J. Microbiol. Methods, 2003, 53, 245-252.

18 S. A. Dunbar, Clin. Chim. Acta, 2006, 363, 71-82.

19 K. N. Vyas, J. J. Palfreyman, D. M. Love, T. Mitrelias and C. H. W. Barnes, Lab Chip, 2012, 12, 5272-5278.
20 J. Palfreyman, D. Love, A. Philpott, K. Vyas, C. Cimorra, T. Mitrelias, C. Barnes, L. Muir, G. Cook and R. Keynes, IEEE Trans. Magn., 2013, 49, 285-295.

21 A. O. Adeyeye, J. A. C. Bland, C. Daboo, J. Lee, U. Ebels and H. Ahmed, J. Appl. Phys., 1996, 79, 6120.

22 W. C. Uhlig and J. Shi, Appl. Phys. Lett., 2004, 84, 759.

23 A. Adeyeye, J. Bland, C. Daboo and D. Hasko, Phys. Rev. B: Condens. Matter Mater. Phys., 1997, 56, 3265-3270.

24 S. Goolaup, N. Singh, A. O. Adeyeye, V. Ng and M. B. A. Jalil, Eur. Phys. J. B, 2005, 44, 259-264.

25 A. Aharoni, J. Appl. Phys., 1998, 83, 3432.

26 D.-L. Sun, J.-H. Gao, X.-Q. Zhang, Q.-F. Zhan, W. He, Y. Sun and Z.-H. Cheng, J. Magn. Magn. Mater., 2009, 321, 27372741.

27 M. Knobel, L. C. Sampaio, E. H. C. P. Sinnecker, P. Vargas and D. Altbir, J. Magn. Magn. Mater., 2002, 249, 60-72.

28 S. Goolaup, A. O. Adeyeye, N. Singh and G. Gubbiotti, Phys. Rev. B: Condens. Matter Mater. Phys., 2007, 75, 144430.

29 G. Gubbiotti, S. Tacchi, G. Carlotti, P. Vavassori, N. Singh, S. Goolaup, A. Adeyeye, A. Stashkevich and M. Kostylev, Phys. Rev. B: Condens. Matter Mater. Phys., 2005, 72, 224413.

30 D. A. Allwood, G. Xiong, M. D. Cooke and R. P. Cowburn, J. Phys. D: Appl. Phys., 2003, 36, 2175-2182.

31 T. A. Moore and J. A. C. Bland, J. Phys.: Condens. Matter, 2004, 16, R1369-R1386.

32 R. Wood, M. Williams, A. Kavcic and J. Miles, IEEE Trans. Magn., 2009, 45, 917-923.

33 K. Vyas, PhD thesis, University of Cambridge, 2013.

34 L. Néel, Ann. Geophys., 1949, 5, 99-136.

35 K. F. Kastl, C. R. Lowe and C. E. Norman, Anal. Chem., 2008, 80, 7862-7869. 